\title{
Mapping Intrinsic Electric Fields Through Off-Axis Electron Holography: Prospects and Problems
}

Falk Röder*, Daniel Wolf*, Axel Lubk*, Matrin Linck*, Karin Vogel*, Andreas Lenk*, Kenji Tsuda** and Hannes Lichte*

* Technical University of Dresden, Institute for Structure Physics, Triebenberg Laboratory, D-01062 Dresden, Germany

** Center for Advanced Microscopy and Spectroscopy, Institute for Multidisciplinary Research for Advanced Materials, Tohoku University, Sendai 980-8577, Japan

The off-axis electron holography approach provides access to phase modulation of the object transmitting electron wave in an electron microscope. The phase is essential more sensitive for electric and magnetic fields in and around the sample than the amplitude hence serves as the key observable for mapping such object properties [1]. The measuring of potential-jumps in silicon based semi-conductor devices [2] and polarization in magnetic domains, like in magnetic memory shape alloys (FIG.1), nowadays ranks among standard applications of off-axis electron holography.

As an extension of that 2D analysis technique, we present 3D phase reconstructions by means of electron holographic tomography affording direct measurement of the electrostatic potential without the drawbacks of 2D imaging e.g. thickness determination and projection effects [3] (FIG.2). It allows e.g. the precise determination of charge carrier concentration of electrons and holes across pnjunctions at nm scale.

The advancing miniaturization of data storage devices down to physical limits requires a detailed understanding of main influences in switching ferroelectric nano-domains. Thereby, the generation and propagation of domain walls and their electrostatic potential barriers seem to play an important role (e.g. [4]). By means of electron holography we attempt to measure possible potential jumps at those barriers, which hits on the current limitations in signal resolution (FIG.3). We present improved reconstructions techniques, which are suitable to reduce the noise and to use the maximum available spatial resolution.

The phase analysis in a material also requires knowledge about the influence of dynamic scattering of the electron wave due to its strong interaction with the crystal potential. Especially for a $90^{\circ}$ domain wall, we demonstrate grave alterations of the phase shift in presence of dynamic scattering using imaging simulation methods and discuss the efficiency of recently applied methods to suppress these influences.

[1] H. Lichte and M. Lehmann, Rep. Prog. Phys. 71 (2008) 016102

[2] M. R. McCartney, D. J. Smith, R. Hull, J. C. Bean, E. Völkl, B. G. Frost, Appl. Phys. Lett. 65 (1994) 2603-2605.

[3] D. Wolf, A. Lubk, H. Lichte, and H. Friedrich, In Press, Corrected Proof, Ultramicroscopy (2010), doi:10.1016/j.ultramic.2009.12.015

[4] C.-L. Jia, S.-B. Mi, K. Urban, I. Vrejoiu, M. Alexe and D. Hesse, Nat. Mat. 7, 57 - 61 (2008)

[5] We acknowledge financial support from the European Union under the Framework 6 program under a contact for an Integrated Infrastructure Initiative. Reference 026019 ESTEEM. 


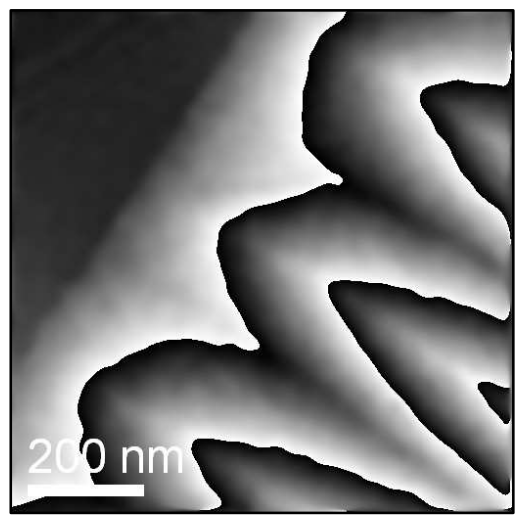

A

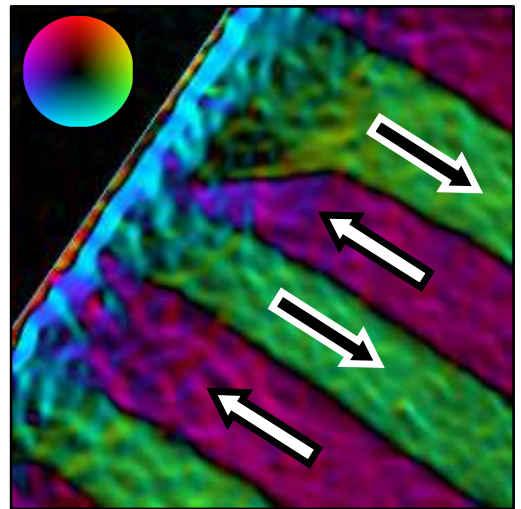

B

FIG. 1. (A) 2D phase image of $180^{\circ}$ magnetic domains in $\mathrm{Ni}_{50} \mathrm{Mn}_{30} \mathrm{Ga}_{20}$ memory shape alloy influenced by phase wrapping and thickness increase of the sample. (B) Thickness corrected gradient image of the unwrapped phase image. Color wheel indicates projected magnetization direction of neighbored domains. Specimen provided by Dr. Stephan Roth, IFW Dresden Germany.
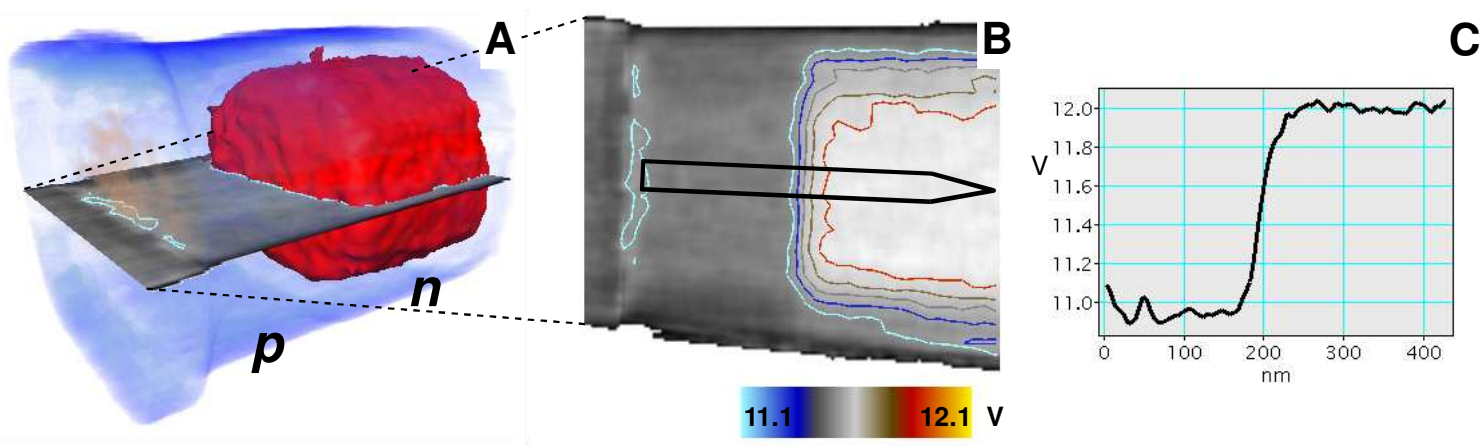

FIG. 2. 3D-reconstruction of the electrostatic potential distribution of pn-doped silicon needle. (A) Discrimination between $\mathrm{p}$ and $\mathrm{n}$ - doped region in top of the needle through $3 \mathrm{D}$ rendered $11.1 \mathrm{~V}$ isosurface (red) indicating the n-doped part. (B) 2D plane cut exhibiting potential jump pronounced in needle center and flattened at the edge (dead layer effect). (C) Line profile across the junction measures diffusion voltage and mean inner potential of about $1.0 \mathrm{~V}$ and $11.5 \mathrm{~V}$ respectively.

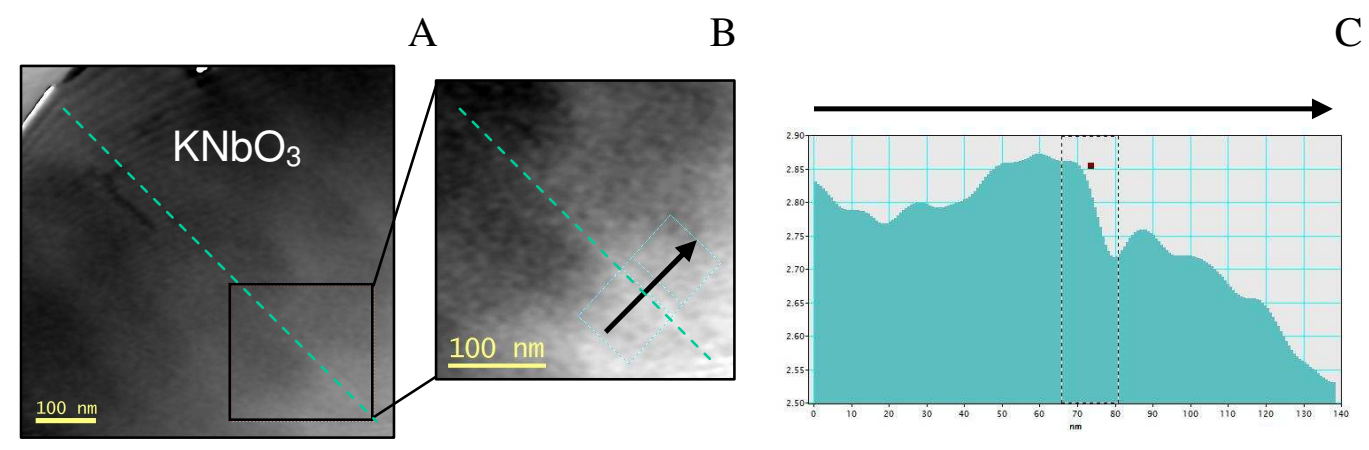

FIG. 3. (A) Phase image of $\mathrm{KNbO}_{3}$ with $90^{\circ}$ domain boundaries (green line) tilted edge-on in kinematic conditions. (B) Magnified phase image with hardly visible domain boundary. (C) Mean line profile exhibits phase jump around $0.1 \mathrm{rad}$ due to potential barrier and/or dynamical scattering. 